\title{
Direct and indirect effects of predation and parasitism on the Anopheles gambiae mosquito
}

\author{
Fedinand Ong'wen, Patrick Ogola Onyango and Tullu Bukhari ${ }^{*}$
}

\begin{abstract}
Background: A good understanding of mosquito ecology is imperative for integrated vector control of malaria. In breeding sites, Anopheles larvae are concurrently exposed to predators and parasites. However, to our knowledge, there is no study on combined effects of predators and parasites on development and survival of larvae and their carry-over effects on adult survivorship and susceptibility to further parasite infection.

Methods: This study focused on effects of the nymphs of the dragonfly Pantala flavescens and the parasitic fungus Beauveria bassiana on Anopheles gambiae, to determine: predation efficacy of nymphs against An. gambiae larvae; development rate of An. gambiae larvae in the presence of one, two or four constrained nymphs; efficacy of B. bassiana against An. gambiae larvae at doses of 3, 6 and $12 \mathrm{mg}$; and survival of adult mosquitoes exposed to B. bassiana, following pre-exposure to a constrained predator and/or parasite at the larval stage. The experiments consisted of survival bioassays quantified as pupation day, or dead larvae and/or adults.

Results: Nymphs had an average predation efficacy of $88.3 \%$ (95\% Cl: 87.5-89.1) at 24 hours, against An. gambiae larvae. The presence of one or two nymphs reduced development time of larvae by 0.65 and 0.35 days, respectively. However, development time of larvae exposed to four nymphs was similar to the control larvae. Larvae exposed to 3, 6 and $12 \mathrm{mg}$ of B. bassiana were 2.0,2.5 and 3.5 times more likely to die, respectively, compared to control larvae. Adults not pre-exposed, those pre-exposed to predator, parasite, or both were 45.8, 67.4, 50.9 and 112.0 times more likely to die, respectively, compared to control that were unexposed to predator or parasite, at larval and adult stage.

Conclusions: This study shows that both predator and parasite can reduce larval population of An. gambiae, and presence of predator cues decreases development time in breeding sites, as well as, increases the susceptibility of emerging adult to fungus. Predator and parasite both have an additive effect on survival of adults exposed to $B$. bassiana. Field studies are required for an in-depth understanding of predator and parasite influence on mosquito development time, survival and susceptibility in nature.
\end{abstract}

Keywords: Predator, Parasite, Anopheles, Susceptibility, Malaria

\section{Background}

In 2017, the World Health Organization (WHO) estimated 219 million cases and 435,000 deaths due to malaria. This is 20 million less than the number of

*Correspondence: tbukhari@maseno.ac.ke

Department of Zoology, School of Physical and Biological Sciences,

Maseno University, Maseno, Kenya malaria cases in 2010. However, data show that from 2015-2017 there has been no significant reduction in global malaria cases [1]. Human malaria is caused by Plasmodium spp. transmitted by Anopheles mosquitoes. Current malaria mosquito control strategies mainly rely on insecticides and target female Anopheles mosquitoes indoors. Outdoor malaria transmission, insecticide resistance and non-target effects challenge current mosquito 
control programmes [2]. There is, therefore, an increased interest in novel mosquito control strategies and a general understanding that integrated vector management (IVM) is required for a sustainable and environmentally friendly mosquito control programme $[2,3]$.

A good understanding of mosquito ecology is a precondition for IVM to eliminate and eradicate the malaria mosquito [4,5]. Anopheles larvae are aquatic and are found in a variety of breeding sites in terms of size, permanence, vegetation and water cleanliness. Studies have shown that there is high larval mortality in the natural breeding sites due to climatic conditions, parasitism and predation [6-8]. This high mortality can exert a high selection pressure in natural habitats and modify how the emerging adults are equipped for future climate, parasite and predator challenges in their terrestrial environment. It is known that a high proportion of the $A n$. gambiae female population is resistant to infection with Plasmodium parasites and therefore does not contribute to malaria transmission. This resistance is genetic and linked to the immune response of the mosquitoes [9]. Non-genetic factors, e.g. climate, predators and parasites can also influence the resistance of mosquitoes to parasites [10]. Predation (presence of predation reduces phenoloxidase activity) and climatic conditions (appropriate environmental temperature can favor the immune response) indirectly influence the immune system while parasites can directly or indirectly influence the immune system $[11,12]$. These indirect non-lethal effects of both parasites and predators can influence the fecundity, survival and immune response of the surviving individuals and consequently malaria transmission [13-17]. A study conducted by Bargielowski and Koella [13], showed that infection at the larval stage with the microsporidian Vavraia culicis increased the resistance of adult Anopheles mosquitoes to Plasmodium infection. However, An. stephensi females exposed to the fungus Beauveria bassiana at the larval stage and re-exposed to the same fungus during the adult stage had equal survival as females exposed only during the adult stage showing no influence of larval exposure on adult susceptibility [18]. Another study showed that An. gambiae larvae exposed to the entomopathogenic fungus Aspergillus parasiticus developed into adults that had reduced longevity and fecundity [15]. Roux et al. [16] observed that exposure to the predator backswimmer Anisop jaczewskii did not influence the susceptibility of An. coluzzii to Plasmosium falciparum but increased the larval development time, reduced the female wing size as well as influenced the fecundity and longevity. Similarly, Chobua et al. [19] showed that development time of An. gambiae larvae increased in the presence of the predator fishes Carassius auratus and
Gambusia affinis. Also, the presence of predator cues reduced oviposition and resulted in the emergence of more female mosquitoes compared to male [19].

To date, studies on this subject have not looked at the combined effect of predators and parasite presence during the larval stage $[13,15,16]$. In nature, however, the larvae can be concurrently exposed to both predators and parasites. This study reports the lethal and nonlethal effects of both predation and parasitism on the larval stage of An. gambiae and the carry-over effects of these factors on the susceptibility of the adult mosquitoes to a subsequent exposure to the same parasite. In this study we used the dragonfly Pantala flavescens and the entomopathogenic fungus $B$. bassiana as the predator and parasite of An. gambiae, respectively.

The dragonfly $P$. flavescens is widespread (except in Antarctica), although rare in Europe, and the nymph has been associated with low densities or absence of Anopheles larvae in otherwise suitable breeding sites [20]. Also, in the laboratory $P$. flavescens nymphs were found to be efficient predators with one nymph feeding on $54 \pm 5$ Aedes aegypti larvae within 24 hours [21]. Since 2003, entomopathogenic fungi have gained a renewed interest for their potential to control malaria mosquito larvae and adults [22-26]. Many laboratory and field studies have been conducted focusing on improving the delivery of fungal spores to the mosquitoes and the potential to control insecticide-resistant mosquitoes [22, 24, 27-29]. Multiple strains of Metarhizium anisopliae and B. bassiana have been intensively studied in this regard [18, 22-30].

The main objective of this study was to investigate the direct and indirect effects of the nymphs of the dragonfly $P$. flavescens and the parasitic fungus $B$. bassiana on the development and survival of An. gambiae larvae, as well as the susceptibility to the fungus of mosquito adults, that developed from the larvae exposed to P. flavescens and/ or $B$. bassiana. The specific objectives were to determine: the predation efficacy of $P$. flavescens nymphs against $A n$. gambiae larvae; the development rate of An. gambiae larvae reared in the presence of varying densities of constrained $P$. flavescens nymphs; the efficacy of $B$. bassiana against An. gambiae larvae at doses of 3, 6, and $12 \mathrm{mg}$; and the survival of adult mosquitoes that emerged from water with the predator $P$. flavescens nymph, with the parasite $B$. bassiana, or with both predator and parasite following a second exposure to B. bassiana at the adult stage.

\section{Methods}

Mosquito rearing and experiments were performed at the Animal House Insectary, Department of Zoology in Maseno University, Kenya, from January 2017 
to April 2017. During this period, room temperature ranged between $26-32{ }^{\circ} \mathrm{C}$ and relative humidity ranged between $65-70 \%$.

\section{Anopheles gambiae rearing}

Anopheles gambiae eggs were obtained from the Kenya Medical Research Institute, Kisian (courtesy of $\mathrm{Dr}$ Andrew Githeko). Eggs were added to 1 liter of dechlorinated tap water in plastic bowls $(20 \times 15 \times 5 \mathrm{~cm})$, lined with No. 1 Whatman filter paper to prevent eggs from adhering to the sides of the plastic bowls and drying out.

The hatched larvae were fed on Liquifry No. 1 (Interpet Ltd., Dorking, Surrey, UK) (1 g daily) for the first two days and then on ground cat food (Purina, Go cat ${ }^{\circledR}$, UK) for the rest of the larval period [31]. The developing pupae were removed with a plastic pipette and placed in $300 \mathrm{ml}$ clear plastic cups inside holding cages $(30 \times 30 \times 30 \mathrm{~cm})$ for the adults to emerge. All adults were fed on $6 \%$ glucose soaked in cotton wool.

\section{Pantala flavescens nymph rearing}

Pantala flavescens dragonfly nymphs were captured from rice fields in the Ahero Irrigation Scheme, Ahero, Kenya and identified according to Paul and Kakkassery [32]. Two hundred nymphs were collected and transported to the Animal House Insectary, at Maseno University. Nymphs that weighed below $2.5 \mathrm{~g}$ each were kept together in bowls $(20 \times 15 \times 5 \mathrm{~cm})$ with $250 \mathrm{ml}$ water. Five nymphs were kept in each bowl until they weighed $2.5 \mathrm{~g}$ or more. Nymphs were fed on 100 third-instar larvae in the larval bowls (the average daily number of larvae (20 larvae) fed on by each nymph below $2.5 \mathrm{~g}$ had been pre-determined earlier). Each nymph, that weighed 2.5 g or above, was kept, separately, in a $300 \mathrm{ml}$ clear plastic cup containing $50 \mathrm{ml}$ dechlorinated tap water. The nymphs were fed on 13-14 third-instar mosquito larvae per day, which is half their daily larval consumption, aimed at keeping them alive and active in predation. The uneaten larvae were removed from the cups or bowls once they pupated. Any nymph used in an experiment had to weigh $3 \mathrm{~g} \pm 1 \mathrm{mg}$. Each nymph was starved for 24 $\mathrm{h}$ prior to each experiment.

\section{Beauveria bassiana spores}

Spores of B. bassiana (Strain GHA Technical Active Ingredient, CAS 63428-82-0, Lam International Corporation, Butte, USA) were stored in an airtight plastic container at $4{ }^{\circ} \mathrm{C}$. The germination percentage of spores was tested prior to the experiments and only the spores with $>85 \%$ germination were used [28].

\section{Predation efficacy of $P$. flavescens nymph against $A n$. gambiae larvae}

This experiment had one control group and one treatment group. Each group was replicated four times. Each replicate consisted of 30 third-instar larvae placed in a larval bowl with 1 liter of dechlorinated tap water. In the control replicates, larvae were not exposed to nymphs of $P$. flavescens. In each treatment replicate, larvae were exposed to one $P$. flavescens nymph. In all the groups, the number of surviving larvae was recorded after every hour for the first $12 \mathrm{~h}$, and then, after $24 \mathrm{~h}$.

\section{Development rate of An. gambiae larvae reared in the presence of varying densities of constrained $P$. flavescens nymphs}

This experiment consisted of four groups: one control and three treatment groups. In the control group, larvae were not exposed to predators. In the treatment groups, larvae were exposed to one, two or four P. flavescens nymphs. Each nymph was constrained in a separate clear, plastic cup with small holes (small enough to allow water circulation but no passage to the larvae), in the larval bowl with An. gambiae larvae (Fig. 1a, b). Each nymph was fed on 13 larvae per day. Each group was replicated four times with each replicate consisting of 30 first-instar (1-day-old) larvae placed in the larval bowl with 1 liter of water and observed till pupation day. Development time was recorded as number of days for the larva to develop into a pupa.

\section{The efficacy of $B$. bassiana against An. gambiae larvae}

The efficacy of B. bassiana against An. gambiae larvae was determined by recording the number of dead mosquito larvae after fungal exposure. There were four groups: one control and three treatment groups. Each group had four replicates of 30 first-instar larvae in a bowl with 1 liter of

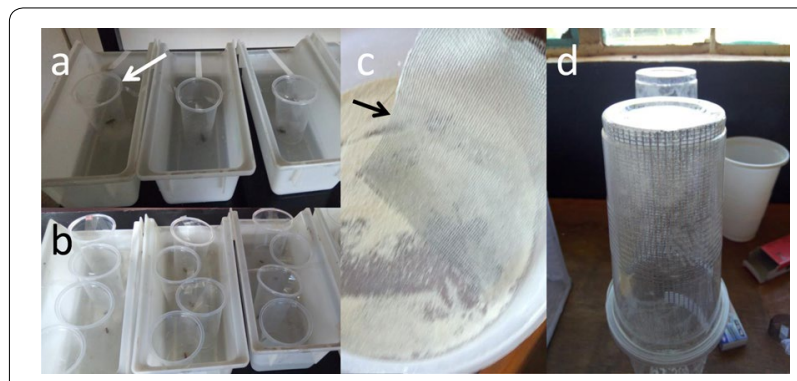

Fig. 1 Bioassay set-up. a Exposure of Anopheles gambiae larvae to one constrained Pantala flavescens in plastic cups (arrow) inside the larval bowl. $\mathbf{b}$ Exposure of An. gambiae larvae to four constrained $P$. flavescens kept in separate plastic cups. c Electrostatic net (arrow) treated by B. bassiana, used for exposing adult An. gambiae to fungus spores. $\mathbf{d}$ Exposure cup with the B. bassiana-treated electrostatic net inside 
water. The control group was not exposed to the fungus, but the treatment groups were exposed to $3 \mathrm{mg}, 6 \mathrm{mg}$ or $12 \mathrm{mg}$ of fungal spores. Fungal spores were dusted on the water surface in the larval bowls. The number of dead larvae was recorded daily until all larvae had either died or pupated. The larvae were fed during the experiment with ground cat food (as mentioned above) and the amount of food was adjusted based on the daily mortality [23].

\section{Survival of adult mosquitoes exposed to fungus $B$. bassiana after predator and/or parasite larval pre-exposure}

This experimental set up comprised of one control group and four treatment groups: Group I, no larval or adult exposure (control); group II, no larval exposure and adults exposed to fungus; Group III, larvae exposed to constrained predator and adults exposed to fungus; Group IV, larvae exposed to fungus and adults exposed to fungus; and Group V, larvae exposed to both predator and fungus and adults exposed to fungus.

\section{Larval infection}

It was determined from the experiments of the second and third objectives that 2 dragonfly nymphs and $6 \mathrm{mg}$ of fungus spores reduced the survival of the An. gambiae larvae but allowed some to pupate. Two nymphs were each constrained separately in a $300 \mathrm{ml}$ clear plastic cup with small holes, which were placed in the same larval bowl that contained the larvae. These nymphs were fed on 13-14 mosquito larvae every day. Larvae were exposed to $6 \mathrm{mg}$ fungus as described earlier. Dead larvae and pupae were separately recorded and removed daily in all the groups. To reach the minimum of 30 adult female mosquitoes per replicate for adult exposure to fungus, the number of larvae exposed to fungus was increased to adjust for the expected larval mortality in treatments. This was based on the results of a previous experiment. However, to ensure that there was no effect of density on larval development, the larvae were exposed to fungus in groups of 30 larvae per tray [23]. In total 15 trays were used to rearing the larvae.

The developing pupae were placed in $300 \mathrm{ml}$ transparent plastic cups, which were covered with a mosquito net. Cotton wool soaked in $6 \%$ glucose solution was put on the net. The females that developed from these pupae were kept in holding cages according to their group.

\section{Adult infection}

Only 2-3-day-old females were exposed to fungal infection. Each of the five groups had three replicates, with 30 females. In the treatment group the females were infected with B. bassiana fungus using electrostatic net (Fig. 1c). The net was cut into $22 \times 11 \mathrm{~cm}$ size pieces and placed in plastic bowl that contained $5 \mathrm{~g}$ fungal spores. The bowl was covered with its lid, shaken thoroughly and allowed to rest for $5 \mathrm{~min}$ for the fungal spores to settle down. The net, now full of fungal spores was removed and fixed inside a $300 \mathrm{ml}$ cup with staple pins to construct the exposure cup (Fig. 1d). The spore density on the electrostatic nets that the adult mosquitoes were exposed to was $3.1 \times 10^{8}$ spores $/ 242 \mathrm{~cm}^{2}\left(1.28 \times 10^{6}\right.$ spores $\left./ \mathrm{cm}^{2}\right)$. The method used to calculate spore density is described in Additional file 1: Text S1. One female mosquito, at a time, was aspirated from the holding cage and introduced into the exposure cup with the fungus-exposed electrostatic net (or for the control, only the electrostatic net) [27]. The top of the cup was covered with mosquito net and the female was left inside for $10 \mathrm{~min}$. After that, the female mosquito was transferred into a holding cup containing $6 \%$ glucose solution-soaked cotton wool and monitored daily till death. The procedure was repeated for all the 360 female adult mosquitoes in the treatment groups.

Dead females were removed from their holding cups and confirmed for fungal infection. This was achieved by dipping cadavers in $70 \%$ ethanol to remove external microbiota (which does not affect the internally growing fungus) [29], then incubated on moist filter paper in sealed Petri dishes at $27 \pm 1{ }^{\circ} \mathrm{C}$. After $3-5$ days, mosquito cadavers were examined for fungal sporulation, specifically emerging hyphae, using a dissecting microscope [25].

\section{Statistical analysis}

To determine the predation efficacy of $P$. flavescens nymphs against $A n$. gambiae larvae, the lethal median time to death (LT50) was calculated by Probit analysis of larval survival over time. Mann-Whitney U-test was used to determine the difference in the mean number of dead (missing) larvae in the control and treatment groups after 24 h. Kaplan-Meier (KM) pairwise comparison was used to determine the difference in development rate (from first-instar larvae to the pupal stage) among An. gambiae larvae reared in the presence of varying densities of $P$. flavescens nymphs. Cox regression was used to determine difference in the likelihood of pupation (the event) in the control and treatment groups in terms of hazard ratio (HR) [33]. The efficacy of varying dosage of B. bassiana against An. gambiae larvae was also determined by Cox regression. In the experiments on survival of adult mosquitoes exposed to the fungus after pre-exposure to predator and/or parasite, LT50 (95\% CI) was calculated by $\mathrm{KM}$ analysis of adult survival over time and $\mathrm{KM}$ pairwise comparison was used to compare the groups. Cox regression was used to determine the difference in survival rates of adult mosquitoes in the control and treatment groups. To determine if there was synergy between predator and 
parasite exposure, mortality rates of the combination of predator and parasite (observed) were compared with the sum of mortalities induced by each, predator and parasite, separately (expected). The expected mortality was calculated using the formula $\mathrm{Mexp}=\mathrm{Mb}+\mathrm{Mp}$ $(1-\mathrm{Mb} / 100)$, where $\mathrm{Mb}$ and $\mathrm{Mp}$ are the observed percent mortalities caused by the parasite and the predator alone, respectively. The expected and observed mortalities were compared using a paired samples t-test [29]. SPSS version 17 was used for all statistical analyses.

\section{Results}

\section{Predation efficacy of $P$. flavescens nymphs} against An. gambiae larvae

No larval mortality was recorded in the control group within $24 \mathrm{~h}$ (no carcasses or injured larvae were found). In contrast, there was high larval mortality in the treatment group within the first hour (Fig. 2), with an average mortality of $88.3 \%$ (95\% CI: $87.5-89.1 \%$ ) after $24 \mathrm{~h}$. In the treatment group, the LT50 calculated by Probit analysis (Slope $\pm \mathrm{SE}=-1.74 \pm 0.48, d f=11, \quad P<0.001$ ) was 0.6 (95\% CI: 0.25-1.0) h. There was a significant difference in the mean number of missing larvae between the control and treatment groups at $24 \mathrm{~h}$ (Mann-Whitney U-test, $\left.Z=-12.667, U=1140, n_{1}=n_{2}=120, P<0.001\right)$ (Fig. 2).

\section{Development rate of An. gambiae larvae reared in the presence of varying densities of constrained P. flavescens nymph}

Varying the density of the constrained predator had a variable effect on the development rates of An. gambiae larvae over time. Larval mortality was recorded as: $5 \%$ (95\% CI: $4.5-5.5 \%)$ in the control group; $2.5 \%$ (95\% CI: $2.1-2.9 \%$ ) in the group exposed to one nymph;

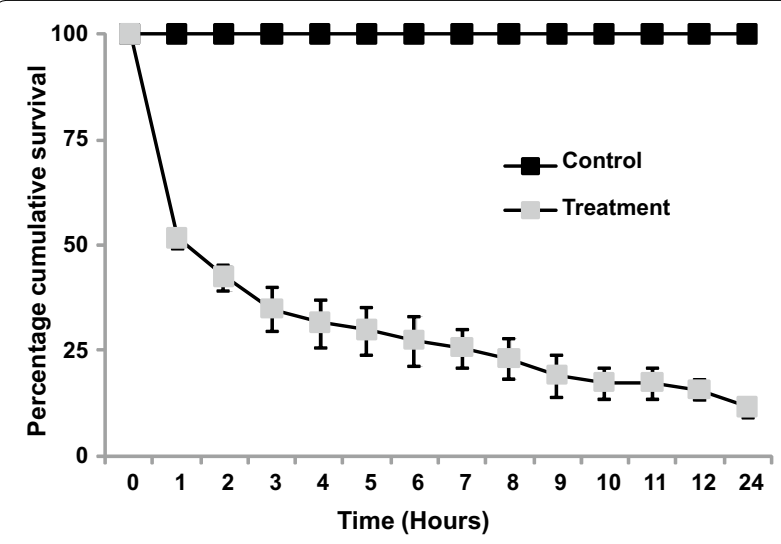

Fig. 2 Percentage cumulative survival ( \pm standard error, SE) of Anophes gambiae larvae over 24 hours. Control group larvae were not exposed to Pantala flavescens nymphs while the treatment larvae were exposed to one free $P$. flavescens nymph
9.2\% (95\% CI: 8.9-9.4\%) in the group exposed to two nymphs; $5.8 \%$ (95\% CI: 5.5-6.2\%) in the group exposed to four nymphs. The surviving larvae in the control group took the longest period, a mean of 7.85 (95\% CI: 7.8-8.0) days, to develop to pupae. When larvae were exposed to only one nymph, the mean number of days to pupation was 7.1 (95\% CI: 7.0-7.2), which was the shortest development period across all the groups. Larvae exposed to two nymphs had a mean of 7.4 ( $95 \% \mathrm{CI}$ : 7.3-7.5) days to pupation, while larvae exposed to four nymphs had a mean of 7.75 (95\% CI: 7.6-7.9) days to pupation (Fig. 3). Kaplan-Meier pairwise comparison showed a significant difference in number of days to pupation between control group larvae and the larval groups exposed to one $\left(\chi^{2}=61.5, P<0.001, n=120\right)$ and two nymphs $\left(x^{2}=14.1, P<0.001, n=120\right)$, but no difference between the control group larvae and the larval group exposed to four nymphs $\left(X^{2}=1.5, P<0.23\right.$, $n=120$ ). The number of days to pupation in the larval group exposed to four nymphs was significantly different from that in the larval groups exposed to one $\left(\chi^{2}=54.6, P<0.001, n=120\right)$ and two nymphs $\left(\chi^{2}=8.2\right.$, $P<0.01, n=120$ ) (Fig. 3).

Hazard ratio (HR) values obtained from Cox regression analysis showed that, compared to the larvae in the control group, larvae exposed to one nymph were twice $[\mathrm{HR}=2$ (95\% CI: $1.5-2.6) ; P<0.0001, d f=1]$ as likely to pupate while larvae exposed to two nymphs were 1.4 times $[\mathrm{HR}=1.4(95 \% \mathrm{CI}: 1.1-1.8) ; P<0.02, d f=1]$ more likely to pupate (Table 1 ). However, larvae exposed to four nymphs were as likely to pupate as the control group $[\mathrm{HR}=1.1$ (95\% CI: $0.8-1.4) ; P<0.50, d f=1]$.

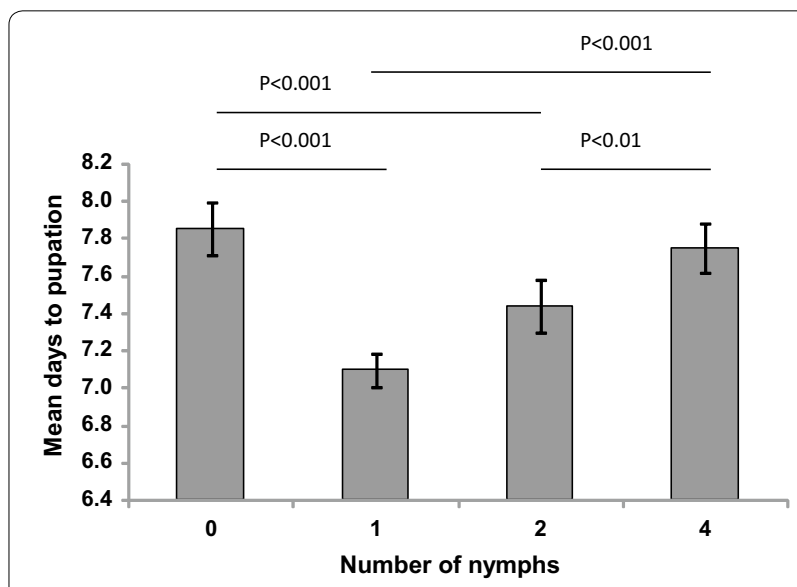

Fig. 3 Mean days to pupation ( \pm standard error, SE) of Anopheles gambiae larvae exposed to constrained 0, 1,2 or 4 Pantala flavescens nymphs. The groups that are significantly different (Kaplan-Meier pairwise comparison) are indicated with $P$-values 
Table 1 Hazard ratios (HR) (event:pupation) of An. gambiae larvae exposed to varying numbers of constrained $P$. flavescens nymphs

\begin{tabular}{llllll}
\hline $\begin{array}{l}\text { No. of } \\
\text { nymphs }\end{array}$ & $\mathrm{HR}$ & $95 \% \mathrm{Cl}$ & $P$-value & $n$ & $d f$ \\
\hline 0 & 1 & - & - & 120 & 3 \\
1 & 2.0 & $1.5-2.6$ & $<\mathbf{0 . 0 0 0 1}$ & 120 & 1 \\
2 & 1.4 & $1.1-1.8$ & $\mathbf{0 . 0 2}$ & 120 & 1 \\
4 & 1.1 & $0.8-1.4$ & 0.50 & 120 & 1 \\
\hline
\end{tabular}

Note: Significant $P$-values (Cox regression) are shown in bold

Abbreviations: $n$, sample size; $d f$, degrees of freedom

\section{Efficacy of varying dosage of B. bassiana against An. gambiae larvae}

The germination percentage of fungus spores used for this experiment was 97\%. All treatment (fungus-exposed) groups had lower survival than the control group (un-exposed) and there was a clear doseresponse (Fig. 4). Cox regression analysis showed a significant difference between the survival of larvae in the control group and all larvae in the treatment groups exposed to $3 \mathrm{mg}, 6 \mathrm{mg}$ and $12 \mathrm{mg}$ of fungus. The analysis showed that, compared to the control group, larvae exposed to $3 \mathrm{mg}$ of fungus were twice as likely to die $[\mathrm{HR}=2.0$ (95\% CI: $1.2-3.3) ; P=0.01, d f=1]$ than the control larvae, while those exposed to $6 \mathrm{mg}$ and $12 \mathrm{mg}$ of fungus were $2.5[\mathrm{HR}=2.5$ (95\% CI: $1.5-4.2)$; $P<0.0001, d f=1]$ and $3.5[\mathrm{HR}=3.5$ (95\% CI: $2.2-5.7)$; $P<0.0001, d f=1]$ times, respectively, more likely to die compared to the control larvae (Table 2).

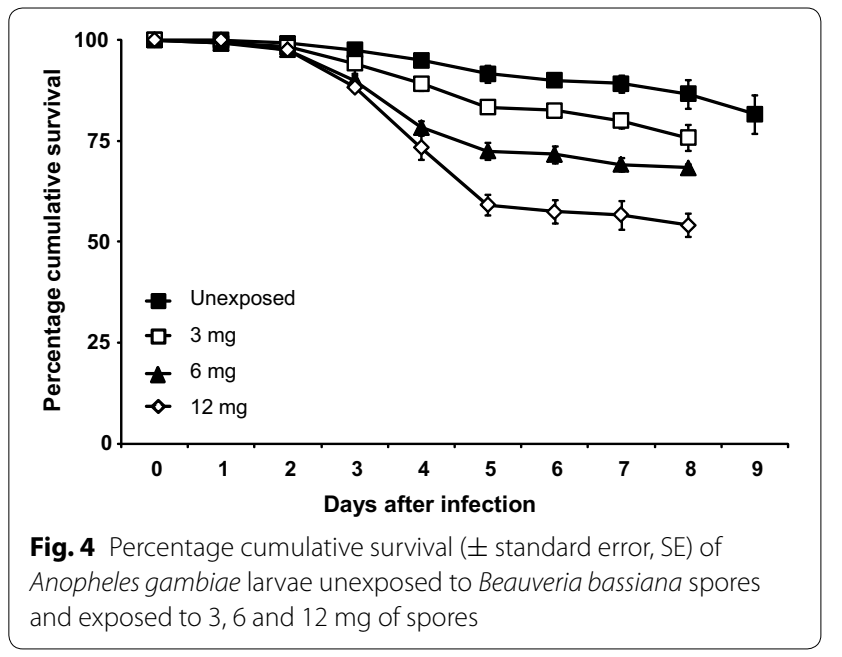

Table 2 Hazard ratios (HR) of An. gambiae larvae exposed to varying doses of $B$. bassiana spores

\begin{tabular}{llllll}
\hline $\begin{array}{l}\text { Fungal dose } \\
(\mathrm{mg})\end{array}$ & $\mathrm{HR}$ & $95 \% \mathrm{Cl}$ & P-value & $n$ & $d f$ \\
\hline 0 & 1 & - & - & 120 & 3 \\
3 & 2.0 & $1.2-3.3$ & $\mathbf{0 . 0 1}$ & 120 & 1 \\
6 & 2.5 & $1.5-4.2$ & $\mathbf{< 0 . 0 0 0 1}$ & 120 & 1 \\
12 & 3.5 & $2.2-5.7$ & $<\mathbf{0 . 0 0 0 1}$ & 120 & 1 \\
\hline
\end{tabular}

Notes: Significant $P$-values (Cox regression) are shown in bold

Abbreviations: $n$, sample size; $d f$, degrees of freedom

Survival of adult mosquitoes exposed to fungus B. bassiana after predator and/or parasite larval pre-exposure

Kaplan-Meier analysis of survival data on adult mosquito exposure to fungus following larval pre-exposure to predator and/or fungus indicate that control group adults had an LT50 of 23 days, which is longer than the LT50 of all treatment groups (Table 3). All mosquito cadavers in the treatment groups were positive for fungal infection (hyphae observed under the microscope), while mosquito cadavers from the control group were negative.

Considering the lethal median time (LT50), control group adults (Group I) survived longer than adults not pre-exposed to any factor during the larval stage (Group II), adults pre-exposed to predator (Group III), adults pre-exposed to fungus (Group IV) and adults preexposed to both predator and fungus (Group V) (Fig. 5).

Hazard ratios indicated that adults in Group V were most likely to die after fungal exposure $[\mathrm{HR}=112$ (95\% CI: 43.3-289.5); $P<0.001, d f=1$ ] followed by Group III $[\mathrm{HR}=67.4$ (95\% CI: 26-174.2); $P<0.001$, $d f=1)$ ]. Kaplan-Meier pairwise comparison showed that exposure to fungus during the larval stage had no effect on the fungus susceptibility at the adult stage, as Groups IV and II were not significantly different $\left(\chi^{2}=1, P=0.316\right.$ ) (Table 4$)$. However, exposure to $P$. flavescens nymph during the larval stage increased the susceptibility to fungus at the adult stage as Groups III and II were significantly different $\left(\chi^{2}=16.7, P<0.001\right)$ (Tables 3, 4). Exposure to only P. flavescens nymph or fungus spores during the larval stage influenced the susceptibility of adults to fungus spores, with mosquitoes exposed to the nymph during the larval stage being more susceptible. This was indicated by the significant difference between Groups III and IV $\left(x^{2}=8.67\right.$; $P=0.003$ ) (Table 4) and Group III showed a higher hazard rate compared to Group IV $[\mathrm{HR}=67.4$ (95\% CI: 26.0-174.2); $P<0.001, d f=1$ versus $\mathrm{HR}=50.9$ (95\% CI: 19.8-130.8); $P<0.001 . d f=1$ ] (Table 3 ). The synergistic effects of predation stress and fungal infection on adult longevity (unexposed to fungal infection) were not 
Table 3 Lethal median time (LT50) and hazard ratios (HR) of An. gambiae adults that emerged from unexposed larvae or larvae exposed to two P. flavescens nymphs and/or $6 \mathrm{mg}$ B. bassiana fungus spores

\begin{tabular}{|c|c|c|c|c|c|c|c|c|c|}
\hline \multirow[t]{2}{*}{ Group } & \multicolumn{2}{|c|}{ Larval exposure to } & \multirow{2}{*}{$\begin{array}{l}\text { Adult exposure to } \\
\text { fungus }\end{array}$} & \multirow[t]{2}{*}{ LT50 (95\% CI) (days) } & \multicolumn{5}{|c|}{ Hazard ratio } \\
\hline & Nymph & Fungus & & & $\mathrm{HR}$ & $95 \% \mathrm{Cl}$ & $P$-value & $n$ & $d f$ \\
\hline | & No & No & No & $23(20-26)$ & - & - & - & 90 & 4 \\
\hline$\|$ & No & No & Yes & $5(4.7-5.3)$ & 45.8 & $17.8-117.7$ & $<0.001$ & 90 & 1 \\
\hline III & Yes & No & Yes & $4(3.8-4.1)$ & 67.4 & $26.0-174.2$ & $<0.001$ & 90 & 1 \\
\hline IV & No & Yes & Yes & $5(4.7-5.3)$ & 50.9 & $19.8-130.8$ & $<0.001$ & 90 & 1 \\
\hline V & Yes & Yes & Yes & $4(3.7-4.3)$ & 112.0 & $43.3-289.5$ & $<0.001$ & 90 & 1 \\
\hline
\end{tabular}

Notes: Significant $P$-values (Cox Regression) are shown in bold

Abbreviations: $n$, sample size; $d f$, degrees of freedom

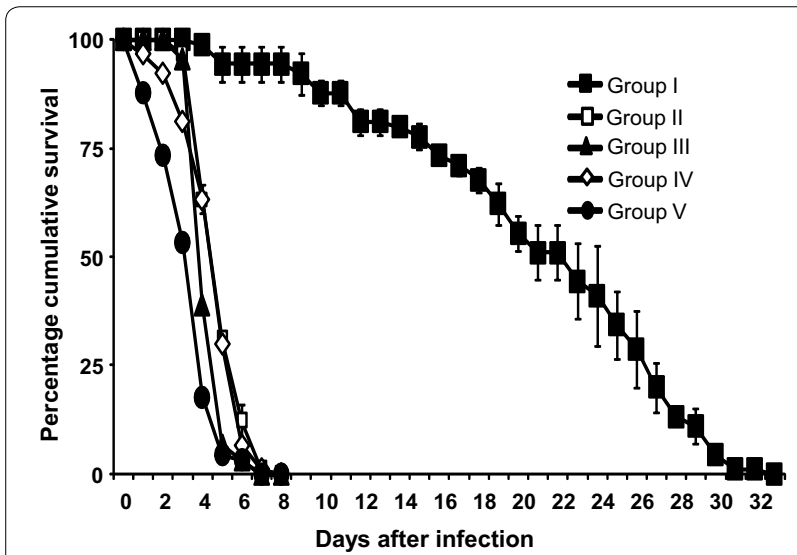

Fig. 5 Percentage cumulative survival ( \pm standard error, SE) of Anopheles gambiae adults. Group I, unexposed; Group II, exposed to Beauveria bassiana fungus at the adult stage; Group III, exposed to Pantala flavescens nymphs at the larval stage and B. bassiana at the adult stage; Group IV, exposed to B. bassiana at the larval and adult stage; Group V, exposed to both P. flavescens nymphs and B. bassiana at the larval stage and $B$. bassiana at the adult stage

significant $(P=0.22)$, despite a mean difference of $6 \%$ in the observed and expected mortality.

\section{Discussion}

The results of this study confirmed the predation efficacy of $P$. flavescens nymphs and virulence of $B$. bassiana spores against $A n$. gambiae larvae. It also confirmed the non-lethal effects of constrained $P$. flavescens nymphs on the development and survival of An. gambiae larvae. Interestingly, the study showed that larval exposure to $P$. flavescens nymphs increased the susceptibility of adults to $B$. bassiana spores, but larval exposure to $B$. bassiana spores did not influence the adult susceptibility to $B$. bassiana spores.

Nymphs of Pantela flavescens have a high predator efficacy against An. gambiae larvae feeding on almost 26 third-instar larvae within 24 hours. High predation efficacy of $P$. flavescens and other species of dragonfly nymphs against An. arabiensis and Aedes aegypti larvae has been reported $[20,21]$. Similarly, high predation efficacy was also reported by Ameka [34], in which thirdinstar larvae of An. gambiae were exposed to nymphs of an unspecified species of dragonfly to determine their predation efficiency. Despite the high predation efficacy reported in the present study, it is important to note that the study set-up was experimental; therefore, it is not clear whether similar predation levels occur in the natural breeding sites and in presence of alternative prey.

Our results show that the effect on the development rate of mosquito larvae under predation risk was

Table 4 Kaplan-Meier pairwise ( $P$-values) comparison of An. gambiae groups

\begin{tabular}{|c|c|c|c|c|c|c|c|c|}
\hline \multirow[t]{2}{*}{ Group } & \multicolumn{2}{|l|}{ I } & \multicolumn{2}{|l|}{ ॥ } & \multicolumn{2}{|l|}{ III } & \multicolumn{2}{|l|}{ IV } \\
\hline & $x^{2}$ & $P$-value & $x^{2}$ & $P$-value & $x^{2}$ & $P$-value & $x^{2}$ & $P$-value \\
\hline$\|$ & 178.6 & $<0.001$ & & & & & & \\
\hline III & 182.0 & $<0.001$ & 16.7 & $<0.001$ & & & & \\
\hline IV & 174.8 & $<0.001$ & 1.00 & 0.316 & 8.67 & 0.003 & & \\
\hline V & 187.6 & $<0.001$ & 50.9 & $<0.001$ & 24.0 & $<0.001$ & 34.8 & $<0.001$ \\
\hline
\end{tabular}

Notes: Anopheles gambiae groups: I, unexposed; II, exposed to Beauveria bassiana fungus at the adult stage; III, exposed to $P$. flavescens nymphs at the larval stage and B. bassiana at the adult stage; IV, exposed to B. bassiana at the larval and adult stage; V, exposed to both P. flavescens nymphs and B. bassiana at the larval stage and $B$. bassiana at the adult stage. Non-significant $P$-values (Kaplan-Meier pair wise comparison) are shown in bold 
dependent on the density of the predator. The development time of An. gambiae larvae reared in the presence of one or two constrained $P$. flavescens nymphs was shorter than that for larvae reared in the absence of $P$. flavescens nymphs. However, the development time of $A n$. gambiae larvae reared in the presence of four constrained P. flavescens nymphs and those reared in the absence of $P$. flavescens nymphs was the same. The reduced development time is in contrast to studies such as Stoks et al. [12] and Beketov et al. [35] showing an increase in the development time of prey under predation risk, but consistent with Culler et al. [37] and Zuhara et al. [36]. In general, threat-sensitive response hypothesis predicts that prey adjust the time spent on antipredator response according to the threat level to allow as much time as possible for foraging and development $[38,39]$. However, the effect of predator on prey development is varied and depends on both the species of prey and predator, as well as on the hunting mode of the predator [39-41]. In the study conducted by Stoks et al. [12] the larvae of damselfly Lestes viridis were exposed to one confined stickleback predator fish Gasterosteus aculeatus. As a result, the prey was exposed to both visual cues and predator kairomones. The stickleback fish has a similar hunting mode to dragonfly nymphs, where it sits, pursues, attacks and captures its prey. However, damselfly larvae are cannibalistic, and the conspecific cues might have also played a role, resulting in slower development time. Beketov et al. [35], unlike in this study, exposed the Culex pipiens mosquito larvae only to the cues of the predator Notonecta glauca that had been fed on Culex larvae or another prey like Daphia magna. The feeding could have resulted in addition of alarm pheromones, produced by the prey before being predated on by N. glauca, into the predator cues of $N$. glauca. This might have resulted in the delayed development of the Culex larvae. It is also known that predator cues produced by a predator that hunts in a sit and pursue mode, like dragonfly nymphs, have a stronger non-lethal effect than a predator that hunts actively like N. glauca [40]. In the study by Culler et al. [37], the predator dytiscid beetle larvae use a variety of hunting modes, including sit-and-wait and active hunting, the former being similar to the dragonfly nymphs [40, 42].

As the number of $P$. flavescens nymphs increased in the bioassays, the development time of the An. gambiae larvae increased so that it was equal to the unexposed larvae in the presence of four nymphs. There can be three explanations for this: (i) cannibalism among the predator; (ii) sensory habituation; and (iii) risk assessment by the prey [43]. The first explanation is cannibalism among the predator. Studies mentioned above were carried out with either one predator in the bioassay or predator cues [12, 35, 37]. Aquatic predators, i.e. backswimmers, diving beetles and dragonfly nymphs are known to be cannibals [44-46]. Dragonfly nymphs have a density-dependent cannibalism and both visual and chemical cues play a role in determining the presence of a conspecific nymph [46, 47]. These cues reduce the foraging activity of the dragonfly nymphs [46]. As the nymph density increased, the chemical cues may have changed releasing the mosquito larvae from the effects of intimidation by the predator [48]. However, in the present study, nymphs of the same weight were used and chemical cues from larger conspecifics has a stronger effect on smaller individuals [46]. The second explanation is the sensory habituation of the larvae to the high concentration of predator cues due to the presence of four nymphs. The high concentration is indicative of imminent attack, which was never experienced due to the fact that the $P$. flavescens nymphs were confined. As a result, the larvae developed sensory habituation that resulted in either a lower anti-predator behavior or the receptors for the specific predator cues stopped responding due to continuous stimulation [43]. The latter is similar to the principle behind "mating disruption" used for pest control in agriculture [49]. The third explanation is risk assessment. In the present experiments, the larvae under low predation risk ( 1 predator) increased their foraging activity to escape the risky larval site, as soon as possible. However, under high predation risk (4 predators), the foraging activity was assessed by the larvae as high risk. Consequently, the larvae adopted a more basal foraging rhythm to be less conspicuous $[38,43,50]$.

Beauveria bassiana fungus negatively affected the survival of An. gambiae larvae and the effect was dose dependent. The control larvae which were not exposed to the fungal parasite survived longer than the treatment larvae. This is consistent with the studies of Bukhari et al. [23] and Vogels et al. [18]. Anopheles larvae hang horizontally to the water surface because of their short siphons and are surface feeders. The spores kill the larvae by either contact with the epicuticle or by being ingested together with food, leading to infection. Spores that enter the larval body through the mouth or siphon also mechanically block these passages [23]. The attached spores germinate, releasing endotoxins as well as damaging the larval tissues during their vegetative growth [51]. These varied modes of action of the fungus lower the probability that resistance will develop against the fungus [23]. The presence of more spores on the water surface increases the likelihood of the larvae coming in contact with the spores and feeding, hence, the observed dosedependent effect $[14,22,23]$. However, at higher doses, the fungus spores tend to clump together due to their hydrophobic nature and the dose-dependent effect is either not observed or is not proportional [23]. 
The survival of adult mosquitoes exposed to B. bassiana fungus after larval pre-exposure to the predator and/or parasite showed that predator presence in the larval breeding sites can influence the susceptibility to parasites in the adult stage. To our knowledge, this is the first study that indicates the influence of predator presence during the larval stage, on the susceptibility to a fungal entomopathogenic fungus parasite at the adult stage of An. gambiae mosquitoes. A study carried out by Yin et al. [52] showed that Daphnia water fleas exposed to fish kairomones were more susceptible to the yeast parasite Metschnikowia. In another study, it was found that above ground predators attacked the juvenile stages of a herbivore beetle Leptinotarsa decemlineata feeding on plant foliage, and made them more susceptible to the below-ground parasites that attacked the pupal stage resulting in high mortality of the beetle [53]. A possible explanation is the trade-off between anti-parasite and anti-predator defenses [16]. Exposure to predators is known to reduce the ability of hosts to cope with parasitism mediated through effects on immune function [54]. Perceived risks of predation and parasitism alters the components of the immune system such as haemocyte density and phenoloxidase activity [55]. However, the results of our study is in contrast to the study of Roux et al. [16], which showed that exposure to the predator backswimmer (Anisops jaczewskii) did not significantly alter mosquito (An. coluzzii) susceptibility to P. falciparum. One explanation can be the active hunting mode of $A$. jaczewskii that led to lower non-lethal effects on the host by reducing the adult size, fecundity and longevity $[16,40]$. Another explanation may be that, in the study of Roux et al. [16], the predator was free to prey upon mosquito larvae which could have suppressed the weakest larvae and selected the strongest. The strong larvae were consequently able to resist several stresses. In our study, body size, survival and fitness of the adult An. gambiae mosquitoes were not considered. Another possible explanation may also be the mosquito innate immunity which possesses a fine-grained capacity to distinguish between classes of closely related eukaryotic parasites, hence, the difference observed in the susceptibility of the adults (pre-exposed to predator during the larval stage) after exposure to B. bassiana and P. falciparum [56, 57].

Parasite presence in the breeding site did not influence the susceptibility to parasite at the adult stage. This result is consistent with Vogels et al. [18] that showed that there was no significant difference in survival of $A$. stephensi females that were only exposed during the adult stage and females that were exposed during both the larval and adult stage. The study by Vogels et al. [18] also showed that only $14 \%$ of the females carried the infection from the larval stage. The similar susceptibility of mosquito adults to parasite in both groups (exposed or unexposed to parasite at the larval stage), is therefore more likely to be due to the low percentage of individuals carrying the infection and not due to an immune response. Indeed, it is noteworthy that the longevity of females that carried the infection from the larval stage was reduced compared to females that were not exposed during the larval stage. The longevity of females that did not carry infection from the larval stage was similar to females that were not exposed during the larval stage. In our study, the same individuals were used for larval and adult exposure, so we were unable to confirm if the infection was carried from the larval stage or was the result of adult exposure. Vogels et al. [18] considered this result an advantage, as control of both larval and adult stages using entomopathogenic fungi can complement each other. As far as the susceptibility to fungus is concerned, the results of our study showed that the presence of both predator and parasite have an additive effect.

The carry-over effects of dragonfly nymph, i.e. increased susceptibility to the fungus, can have an indirect effect on malaria transmission. There are conflicting studies regarding the reduced potential of malaria transmission by Anopheles mosquitoes when co-infected with fungus and Plasmodium during the adult stage $[58,59]$. However, if indeed co-infection with fungus can reduce the potential of adult Anopheles mosquito to transmit malaria, the predator presence during the larval stage may enhance the reduction in malaria transmission [17]. It would be interesting to know if the predators can also increase the susceptibility to transgenic fungi that are actually designed to kill human malaria parasites [60].

Nonetheless, increased susceptibility to B. bassiana can influence malaria transmission by reducing the survival and influencing the behavior of the infected adult mosquitoes [61]. Also, predator presence in breeding sites can complement control programmes that deploy entomopathogenic fungi [30, 62]. This and follow-up studies may be used for informed decision-making on whether a mosquito breeding site needs to be treated or better left untreated when targeting the larval stage. Predators are generally present in large and semi-permanent or permanent breeding sites, e.g. rice paddies. Although easy to locate, they have a large surface area that needs to be treated, which is mired by the vegetation as well as the economic cost [63]. Large breeding sites that harbor predators, like dragonfly nymphs, would therefore be better left untreated to complement a control programme based on entomopathogenic fungi. However, further studies are needed to confirm this.

There are two main points to consider when interpreting the results of our study. First, the experiments were carried out in a laboratory setting with reared $A n$. 
gambiae. Secondly, the predator was constrained. A free swimming and feeding predator may not have the same effect. Whether or not the predator presence can increase the susceptibility of Anopheles adults under natural conditions needs to be investigated.

\section{Conclusions}

Both the parasite B. bassiana and the predator P. flavescens reduced the survival of An. gambiae larvae. Predator presence can also influence the development time of larvae. The surviving adults showed no increase in susceptibility to $B$. bassiana when pre-exposed to the same parasite during larval stage, but an increased susceptibility to $B$. bassiana was observed when adults were pre-exposed to the predator $P$. flavescens during the larval stage. This indicates that in addition to reducing the survival of malaria mosquitoes in the breeding site, the predator may also impact the immune response of the surviving individuals, making them less suitable for disease transmission. However, studies need to be carried out in field conditions for a realistic perspective.

\section{Supplementary information}

Supplementary information accompanies this paper at https://doi. org/10.1186/s13071-020-3915-8.

Additional file 1. Calculation of fungus spore density on electrostatic net.

\section{Abbreviations}

WHO: World Health Organization; IVM: Integrated vector management; HR: Hazard ratio.

\section{Acknowledgments}

We thank Julius Onawa from the Department of Zoology, Maseno University, Kenya, for his technical support, the Kenya Medical Research Institute (Kisian) staff, Dr. Andrew Githeko and Maxwel Gesuge for providing us with the eggs of An. gambiae mosquito (Kisumu strain); IN2CARE ${ }^{\circledR}$, Wageningen, The Netherlands, for providing us with the fungus B. bassiana; George Opito, Daniel Ogwang and Emmanuel Mtata from the National Irrigation Board, Kenya, for their help in the survey and collection of dragonfly nymphs at Bunyala and Ahero Irrigation Schemes.

\section{Authors' contributions}

FO collected and analyzed the experimental data and wrote the first draft of the manuscript. POO was involved in the study design, data analysis and interpretation, and contributed to writing the first draft of the manuscript. TB conceived the study, supervised data analysis and drafted the final version of the manuscript. All authors read and approved the final manuscript.

\section{Funding}

This project was partly funded by National Research Fund (NRF 2017), Kenya.

\section{Availability of data and materials}

Data supporting the conclusions of this article are provided within the article. The datasets used and/or analysed during the present study are available from the corresponding author on reasonable request.

\section{Ethics approval and consent to participate}

Scientific clearance was granted by the School of Graduate Studies, Maseno University, Kenya (MSC/SC/00081/014). Mosquitoes were reared according to the standard protocols at Kenya Medical Research Institute, Kisian. No human subject, human data or human tissue was involved in the study.

\section{Consent for publication}

Not applicable.

\section{Competing interests}

The authors declare that they have no competing interests.

Received: 3 October 2019 Accepted: 24 January 2020

Published online: 30 January 2020

\section{References}

1. WHO. World malaria report 2018. Geneva: World Health Organization; 2018. https://apps.who.int/iris/bitstream/handle/10665/275867/97892 41565653-eng.pdf?ua=1. Accessed 16 Jan 2020.

2. Riveron JM, Tchouakui M, Mugenzi L, Menze BD, Chiang MC, Wondji CS. Insecticide resistance in malaria vectors: an update at a global scale. In: Manguin S, Dev V, editors. Towards malaria elimination-a leap forward. Rijeka: IntechOpen; 2018. p. 149-75.

3. Killeen GF, Tatarsky A, Diabate A, Chaccour CJ, Marshall JM, Okumu FO, et al. Developing an expanded vector control toolbox for malaria elimination. Br Med J Glob Heal. 2017;2:1-8.

4. Ferguson HM, Dornhaus A, Beeche A, Borgemeister C, Gottlieb M, Mulla $M S$, et al. Ecology: a prerequisite for malaria elimination and eradication. PLoS Med. 2010;7:e1000303.

5. Mukabana WR, Kannady K, Kiama GM, ljumba JN, Mathenge EM, Kiche I, et al. Ecologists can enable communities to implement malaria vector control in Africa. Malar J. 2006;14:1-14.

6. Paaijmans KP, Wandago MO, Githeko AK, Takken W. Unexpected high losses of Anopheles gambiae larvae due to rainfall. PLOS ONE. 2007;2:e1146

7. Aniedu I, Mutinga JM, Mutero MC. Vertical estimates of survivorship of larvae and pupae of Anopheles gambiae Giles complex in Baringo District, Kenya. Int J Trop Insect Sci. 1993;14:39-48.

8. Service MW. Mortalities of the immature stages of species B of the Anopheles gambiae complex in Kenya: comparison between rice fields and temporary pools, identification of predators, and effects of insecticidal spraying. J Med Entomol. 1977;13:535-45.

9. Riehle MM. Natural malaria infection in Anopheles gambiae is regulated by a single genomic control region. Science. 2006;312:577-9.

10. Lefevre T, Vantaux A, Dabire KR, Mouline K, Cohuet A. Non-genetic determinants of mosquito competence for malaria parasites. PLoS Pathog. 2013:9:e1003365.

11. Boltaña S, Rey S, Roher N, Vargas R, Huerta M, Huntingford FA, et al. Behavioural fever is a synergic signal amplifying the innate immune response. Proc Biol Sci. 2013;280:20131381.

12. Stoks R, De Block M, Slos S, Doorslaer VW, Rolff J. Time constraints mediate predator-induced plasticity in immune function, condition, and life history. Ecol Soc Am. 2006;87:809-15.

13. Bargielowski I, Koella JC. A possible mechanism for the suppression of Plasmodium berghei development in the mosquito Anopheles gambiae by the microsporidian Vavraia culicis. PLOS ONE. 2009:4:e4676.

14. Bukhari T, Takken W, Koenraadt CJM. Biological tools for control of larval stages of malaria vectors-a review. Biocontrol Sci Technol. 2013;23:987-1023.

15. Nnakumusana ES. Laboratory infection of mosquito larvae by entomopathogenic fungi with particular reference to Aspergillus parasiticus and its effects on fecundity and longevity of mosquitoes exposed to sporal infections in larval stages. Curr Sci. 1985;54:1221-8.

16. Roux O, Vantaux A, Roche B, Yameogo KB, Dabiré KR, Diabaté A, et al. Evidence for carry-over effects of predator exposure on pathogen transmission potential. Proc Biol Sci. 2015;282:20152430.

17. Roux O, Robert V. Larval predation in malaria vectors and its potential implication in malaria transmission: an overlooked ecosystem service? Parasit Vectors. 2019:12:217

18. Vogels CBF, Bukhari T, Koenraadt CJM. Fitness consequences of larval exposure to Beauveria bassiana on adults of the malaria vector Anopheles stephensi. J Invertebr Pathol. 2014;119:19-24. 
19. Chobua M, Nkwengulila G, Mahandeb AM, Mwang'onde BJ, Kweka EJ. Direct and indirect effect of predators on Anopheles gambiae sensu stricto. Acta Trop. 2015;142:131-7.

20. Gouagna LC, Rakotondranary M, Boyer S, Lempérière G, Dehecq JS, Fontenille D. Abiotic and biotic factors associated with the presence of Anopheles arabiensis immatures and their abundance in naturally occurring and man-made aquatic habitats. Parasit Vectors. 2012;5:96.

21. Samanmali C, Udayanga L, Ranathunge T, Perera SJ, Hapugoda M, Weliwitiya C. Larvicidal potential of five selected gragonfly nymphs in Sri Lanka over Aedes aegypti (Linnaeus) larvae under laboratory settings. Biomed Res Int. 2018;2018:8759459.

22. Bukhari T, Takken W, Koenraadt CJM. Development of Metarhizium anisopliae and Beauveria bassiana formulations for control of malaria mosquito larvae. Parasit Vectors. 2011:4:23.

23. Bukhari T, Middelman A, Koenraadt CJM, Takken W, Knols BGJ. Factors affecting fungus-induced larval mortality in Anopheles gambiae and Anopheles stephensi. Malar J. 2010;9:22.

24. Farenhorst M, Farina D, Scholte $\mathrm{E}$, Takken W, Hunt $\mathrm{RH}$, Coetzee $\mathrm{M}$, et al. African water storage pots for the delivery of the entomopathogenic fungus Metarhizium anisopliae to the malaria vectors Anopheles gambiae s.S. and Anopheles funestus. Am J Trop Med Hyg. 2008;78:910-6.

25. Scholte E, Njiru BN, Smallegange RC, Takken W, Knols BGJ. Infection of malaria (Anopheles gambiae s.s.) and filariasis (Culex quinquefasciatus) vectors with the entomopathogenic fungus Metarhizium anisopliae. Malar J. 2003;2:29.

26. Scholte EJ, Knols BGJ, Samson RA, Takken W. Entomopathogenic fungi for mosquito control: a review. J Insect Sci. 2004;4:19.

27. Andriessen R, Snetselaar J, Suer RA, Osinga AJ, Deschietere J, Lyimo IN. Electrostatic coating enhances bioavailability of insecticides and breaks pyrethroid resistance in mosquitoes. Proc Natl Acad Sci USA. 2015;112:12081-6.

28. Farenhorst M, Knols BGJ. A novel method for standardized application of fungal spore coatings for mosquito exposure bioassays. Malar J. 2010;9:27.

29. Farenhorst M, Knols BGJ, Thomas MB, Howard AFV, Takken W, Guessan RN. Synergy in efficacy of fungal entomopathogens and permethrin against West African insecticide-resistant Anopheles gambiae mosquitoes. PLoS ONE. 2010;5:e12081.

30. Knols BGJ, Bukhari T, Farenhorst M. Entomopathogenic fungi as the nextgeneration control agents against malaria mosquitoes. Future Microbiol. 2010;5:339-41.

31. Benedict MQ. MR4: Methods in Anopheles research. Atlanta: CDC; 2007.

32. Paul S, Kakkassery FK. Taxonomic and diversity studies on odonate nymphs by using their exuviae. J Entomol Zool Stud. 2013;1:47-53.

33. Cox DR. Models and life-tables regression. J R Stat Soc Ser B. 1972;34:187-220

34. Ameka CM. Effects of insect growth regulator pyriproxyfen on dragonfly nymphs as predators of Anopheles mosquitoes at Mahanga, Vihiga County, Kenya. Nairobi: MSc thesis, Kenyatta University; 2015.

35. Beketov MA, Liess M. Predation risk perception and food scarcity induce alterations of life-cycle traits of the mosquito Culex pipens. Ecol Entomol. 2007;32:405-10

36. Zuharah WF, Fadzly N, Lester PJ. Lethal and sublethal impacts of predaceous backswimmer Anisops wakefieldi (Hemiptera: Notonectidae) on the life-history traits of the New Zealand mosquito Culex pervigilans (Diptera: Culicidae). J Med Entomol. 2013;50:1014-24.

37. Culler LE, Ayres MP, Virginia RA, Culler LE. In a warmer Arctic, mosquitoes avoid increased mortality from predators by growing faster. Proc Biol Sci. 2015;282:20151549.

38. Helfman GS. Threat-sensitive predator avoidance in damselfish-trumpetfish interactions. Behav Ecol Sociobiol. 1989;24:47-58.

39. Roux O, Diabate A, Simard F. Divergence in threat sensitivity among aquatic larvae of cryptic mosquito species. J Anim Ecol. 2014;83:702-11.

40. Preisser EL, Orrock J, Schmitz OJ. Predator hunting mode and habitat domain alter nonconsumptive effects in predator-prey interactions. Ecology. 2007;88:2744-51

41. Shaalan EA, Canyon DV. Aquatic insect predators and mosquito control. Trop Biomed. 2009;26:223-61.

42. Bukhari T, Knols BGJ. Efficacy of Aquatain ${ }^{\mathrm{TM}}$, a monomolecular surface film, against the malaria vectors Anopheles stephensi and An. gambiae s.s. in the laboratory. Am J Trop Med Hyg. 2009;80:758-63.
43. Ferrari MCO, Elvidge CK, Jackson CD, Chivers DP, Brown GE. The responses of prey fish to temporal variation in predation risk: sensory habituation or risk assessment? Behav Ecol. 2010;21:532-6.

44. Yee DA. Behavior and aquatic plants as factors affecting predation by three species of larval predaceous diving beetles (Coleoptera: Dytiscidae). Hydrobiologia. 2010;637:33-43.

45. Orr BK, Murdoch WW, Bence JR. Population regulation, convergence, and cannibalism in Notonecta (Hemiptera). Ecol Soc Am. 1990;71:68-82.

46. Crespo JG. A review of chemosensation and related behavior in aquatic insects. J Insect Sci. 2011;11:1-39.

47. Van Buskirk J. Density-dependent cannibalism in larval dragonflies. Ecol Soc Am. 1989;70:1442-9.

48. Werner EE, Peacor SD. A review of trait- mediated indirect interactions in ecological communities. Ecology. 2003;84:1083-100.

49. Smart L, Aradottir G, Bruce T. Role of semiochemicals in integrated pest management. In: Abrol DP, editor. Integrated pest managment: current concepts and ecological perspective. San Diego: Academic Press; 2014. p. 93-109.

50. Preisser EL, Bolnick DI, Benard MF. Scared to death? The effects of intimidation and consumption in predator-prey interactions. Ecol Soc Am. 2005;86:501-9.

51. Hegedus DD, Khachatourians GG. The impact of biotechnology on hyphomycetous fungal insect biocontrol agents. Biotechnol Adv. 1995:13:455-90.

52. Yin M, Laforsch C, Lohr JN, Wolinska J. Predator-induced defense makes Daphnia more vulnerable to parasites. Evolution. 2011;65:1482-8.

53. Ramirez RA, Snyder WE. Scared sick? Predator-pathogen facilitation enhances exploitation of a shared resource. Ecology. 2009;90:2832-939.

54. Navarro C, de Lope F, Marzal A, Moller AP. Predation risk, host immune response, and parasitism. Behav Ecol. 2004;15:629-35.

55. Joop G, Rolff J. Plasticity of immune function and condition under the risk of predation and parasitism. Evol Ecol Res. 2014;6:1051-62.

56. Mitri C, Jacques J, Thiery I, Riehle MM, Xu J, Morlais I, et al. Fine pathogen discrimination within the APL1 gene family protects Anopheles gambiae against human and rodent malaria species. PLoS Pathog. 2009;5:e1000576.

57. Riehle MM, Bukhari T, Gneme A, Guelbeogo WM, Coulibaly B, Fofana A, et al. The Anopheles gambiae 2La chromosome inversion is associated with susceptibility to Plasmodium falciparum in Africa. Elife. 2017;6:e25813.

58. Heinig RL, Thomas MB. Interactions between a fungal entomopathogen and malaria parasites within a mosquito vector. Malar J. 2015:14:22

59. Blanford S, Chan BHK, Jenkins N, Sim D, Turner RJ, Read AF, et al. Fungal pathogen reduces potential for malaria transmission. Science. 2009;1638:1638-41.

60. Fang W, Vega-Rodríguez J, Ghosh AK, Marcelo Jacobs-Lorena AK, Leger St RJ. Development of transgenic fungi that kill human malaria parasites in mosquitoes. Science. 2011;331:1074-7.

61. Ondiaka S, Bukhari T, Farenhorst M, Takken W. Effects of fungal infection on the host-seeking behaviour and fecundity of the malaria mosquito Anopheles gambiae Giles. Proc Nethelands Entomol Soc Meet. 2008; p. 121-8.

62. Mnyone LL, Kirby MJ, Lwetoijera DW, Mpingwa MW, Simfukwe ET, Knols $B G J$, et al. Tools for delivering entomopathogenic fungi to malaria mosquitoes: effects of delivery surfaces on fungal efficacy and persistence. Malar J. 2010;9:246

63. Bukhari T, Takken W, Githeko AK, Koenraadt CJM. Efficacy of Aquatain, a monomolecular film, for the control of malaria vectors in rice paddies. PLOS ONE. 2011;6:e21713

\section{Publisher's Note}

Springer Nature remains neutral with regard to jurisdictional claims in published maps and institutional affiliations. 